\title{
Elements of System Programming
}

\author{
Pratik Awari $^{1}$, Prathamesh Salunke ${ }^{2}$, Gaurav Rathod ${ }^{3}$, Himanshu Ghatole ${ }^{4}$, Prof. Swati Patil ${ }^{5}$ \\ Students, Computer Technology, Bharati Vidyapeeth's Institute of Technology, Kharghar, India ${ }^{1,2,3,4}$ \\ Professor, Computer Technology, Bharati Vidyapeeth's Institute of Technology, Kharghar, India ${ }^{5}$
}

\begin{abstract}
In this paper it is listed and explained about various components of system programming and there functions. It tells how the resources are made available to the users to execute the program effectively. System programming solves the problem of central processors which minimize the human efforts. System programming provides various system software. It is basically the conversion of high level programming into low level programming i.e. machine code.
\end{abstract}

Keywords: System Programming, SPR, Compiler, Assembler, Lex, Yacc

\section{INTRODUCTION}

System programming is the activity of programming system software. Systems programming aims to produce software which provide services to other software. It is a low level language but programmer writes it in high level language which is not a computer language. To handle this, Compiler and Interpreter convert high level language into machine language. System programming requires great degree of hardware awareness .It is used for creating Operating System and AAA games etc. The main goal is to achieve efficient use of available resources. System programming allows limited facilities. System programming is a way in which the programmer can interact with the system. Programmer will be able to access the system using System programming. Without system programming there is no another way to interact with system. Therefore it's so important. By using System programming we can produce new software\& software platforms which will help to provide services to other software.

\section{LITERATURE REVIEW}

This paper describes an approach to the maintenance problem of central processors which minimizes the human role. This approach consists of a combination of 1) a programming system which computes the diagnostic program from the design-automation tape, and 2) a novel organization of a part of the controls of an experimental computer.[1]

A programming language specifically designed for embedded systems must be designed to create extremely reliable source code. This paper presents overviews and comparisons of Processes with Multiple Clocks (PMC), FORTH and Embedded Eiffel.[2]

The Java programming language has been widely accepted as a general purpose language for developing portable applications, toolkits, and applets. With so much activity in industry and academia in these user-level areas, is it surprising that Java is also an equally capable systems programming language.[3]

Embedded systems have become ubiquitous and are found in numerous application domains such as sensor networks, medical devices, and smart appliances. Software flaws in such systems can range from minor nuisances to critical security failures and malfunctions. Additionally, the computational power found in these devices has seen tremendous growth and will likely continue to advance. With increasingly powerful hardware, the ability to express complex ideas and concepts in code becomes more important.[4]

\section{COMPONENTS OF SYSTEM PROGRAMMING}

Components of system programming are:
1) Loader
2) Assembler
3) Compiler
4) Macro
5) Interpreter 
Vol. 7, Issue 8, August 2018

1) Loader

Loader plays an important role in system programming. It palaces the program in the main memory and prepare it for execution. Loading program means reading contents of executable file and loading program in the memory and then carryout tasks and prepare them for running, as the loading is complete the operating system starts the program execution by passing control to program code.

\section{2) Assembler}

An assembler is used to convert assembly language program into machine level language. Assembler provides access to Software and Application developers to manage computer hardware and its components. Assembler act as a compiler of assembly language program.

3) Compiler

A Compiler is program used to convert high level programming language to low level programming language. Compilers are a type of translator that translates digital devices and computer languages.

Further compiler is divided into two types:-

i) Cross Compiler: A cross compiler is a compiler which is capable of generating an executable code for other platform where it resides on another platform.

ii) Source to Source Compiler: A source to source compiler accepts an input written in one programming language and produces output for same written in another language.

4) Macro

A macro is a single line abbreviation for group of statements or block of code. Macro allows programmer to write the short part of the program. A macro can be expanded into no. of instructions.

5) Interpreter

In System Programming interpreter is a program which directly executes the program without compiling it to the machine language.

The below program shows the use of all components of system programming:

MACRO

ADDITION \&arg1,\&arg2,\&arg3

ADD A,\&arg1

ADD B,\&arg2

ADD C,\&arg3

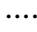

MEND

ADD A, $\arg 1$

ADDITION arg4, arg5, arg6

ADD B, $\arg 2$

..

MEND

Macro expansion can be done using macro call. While in macro expansion the sequence of assembly statement are replaced by macro statement. Here MACRO indicates the beginning of the program and ADDITION is the name of macro. Arguments i.e. \&arg1,\&arg2,\&arg3 are used for the expansion of macro. Set of instruction are used that are added with macro name and dummy parameters. Later macro is been called due to which macro expansion takes place. MEND indicates end of the macro program.

Types of system software are:

i) YACC

ii) LEX

i)YACC: YACC stands for "Yet Another Compiler Compiler" it is used to generate code for parser in C. It is an open source program. The output generated is a shift-reduce parser in C. In yacc file user writes its own main function which calls yyparse() and its end in y.tab.c

ii)LEX: Lex is a computer program which generates lexers or scanners. Lexical analyser reads input stream and produces source code as output. Lex and yacc both work internally. Lex source is a table of regular expressions. The table is translated to a program which reads an input stream and then produces source code as output. 
Vol. 7, Issue 8, August 2018

\section{CONCLUSION}

System programming helps us to get familiar with various system components. It helps to write assembly language programs. SPR consist of various types of system tools like loader, assembler, compiler, interpreter \& macro. It explain different types of system software. It helps us to interact with the system.

\section{REFERENCES}

[1]. K. Maling and E. L. Allen "A Computer Organization and Programming System for Automated Maintenance" https://ieeexplore.ieee.org /document/4038036/authors

[2]. S.S. Maurer "A survey of embedded systems programming languages" https://ieeexplore.ieee.org/document/997974/

[3]. S. Ritchie "Systems programming in Java" https://ieeexplore.ieee.org/document/591652/

[4]. Paul Soulier and Depeng Li "Blueprint for an embedded systems programming language" https://ieeexplore.ieee.org/document/7284225/

[5]. Martin Richards "A tool of compiler writing and system programming" https://dl.acm.org/citation.cfm?id=1476880

[6]. Christian Jacob" Genetic L-System Programming” https://link.springer.com/chapter/10.1007\%2F3-540-58484-6_277

[7]. https://en.wikipedia.org/wiki/System_programming 